\title{
Predico: A System for What-if Analysis in Complex Data Center Applications
}

\author{
Rahul Singh ${ }^{1}$, Prashant Shenoy ${ }^{1}$, Maitreya Natu ${ }^{2}$, \\ Vaishali Sadaphal $^{2}$, and Harrick Vin ${ }^{2}$ \\ 1 Dept. of Computer Science, University of Massachusetts, \\ Amherst, USA \\ \{rahul, shenoy\}@cs.umass . edu \\ 2 Tata Research Development and Design Center, \\ Pune, India \\ \{maitreya.natu, vaishali.sadaphal, harrick.vin\}@tcs.com
}

\begin{abstract}
Modern data center applications are complex distributed systems with tens or hundreds of interacting software components. An important management task in data centers is to predict the impact of a certain workload or reconfiguration change on the performance of the application. Such predictions require the design of "what-if" models of the application that take as input hypothetical changes in the application's workload or environment and estimate its impact on performance.

We present Predico, a workload-based what-if analysis system that uses commonly available monitoring information in large scale systems to enable the administrators to ask a variety of workload-based "what-if" queries about the system. Predico uses a network of queues to analytically model the behavior of large distributed applications. It automatically generates node-level queueing models and then uses model composition to build system-wide models. Predico employs a simple what-if query language and an intelligent query execution algorithm that employs on-the-fly model construction and a change propagation algorithm to efficiently answer queries on large scale systems. We have built a prototype of Predico and have used traces from two large production applications from a financial institution as well as real-world synthetic applications to evaluate its what-if modeling framework. Our experimental evaluation validates the accuracy of Predico's node-level resource usage, latency and workload-models and then shows how Predico enables what-if analysis in two different applications.
\end{abstract}

\section{Introduction}

Today online server applications have become popular in domains ranging from banking, finance, e-commerce, and social networking. Such server applications run on data centers and tend to be complex distributed systems with tens or hundreds of interacting software components running on large server clusters. As an example, consider an online stock trade processing application of a major financial firm. This application consists of 471 separate software components that process incoming stock trades at low latencies. Figure 1 shows another application which disseminates stock prices and market news to the terminals of stock traders; this application consists of 8970 components. 
The components are depicted as nodes of the graph and process stock data and news from a multitude of sources, filter, aggregate, and then disseminate updates for each company to desktops that subscribe to such streams. Such data center applications differ significantly in scale and complexity when compared to traditional multi-tier web applications.

Typical data center applications evolve over time as new functionality is added, its workload volume grows, or its hardware or software is updated. To deal with such changes, an important management task for administrators is to predict the impact of any planned (or hypothetical) change on the performance of individual components or the entire system. This task, which is referred to as what-if analysis, requires the design of what-if models that take as input a potential change in the application workload or its settings and predict the impact of that change on application behavior. However, given the complexity of today's data center application, manual design of such what-if models is no longer feasible since data center administrators may not be able to comprehend the behavior of a complex system of tens of interacting components. Consequently, a what-if analysis system must be able to automatically derive such models from prior observations of application's behavior. Further, the system must be able to scale to large complex applications with hundreds of interacting components, while allowing rich what-if analysis efficiently. While a number of modeling techniques have been proposed for distributed or multi-tier web applications [10|14 17|13]|12], such models are not directly targeted for what-if analysis or are not designed to scale to larger data center applications such as the ones illustrated in Figure 1

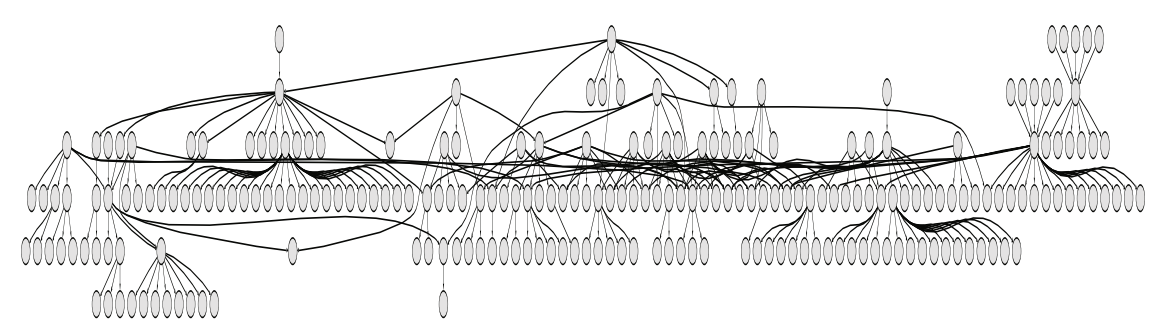

Fig. 1. Stock Price and Market Data Dissemination Application; only a subset of the application is shown for brevity

In this paper, we present Predico, a what-if analysis system to predict the impact of workload changes on the behavior of data center applications. Predico makes the following contributions:

- Modeling of complex data center applications: Predico employs a queuing-theoretic framework to model large distributed data-center applications. Our modeling framework is based on a network of queues and captures the dependence between the workload of each component of the application and the corresponding resource utilization, request latency and the outgoing workloads to other components. Predico uses monitoring data and request $\log$ s to estimate the parameters of such a model 
and employs model composition to create larger system-level models for groups of interacting application components.

- Intelligent query execution: Predico uses a novel change propagation algorithm that uses these models to execute a what-if query and determine the impact of a workload change on other components. This algorithm first computes an influence graph to determine which application components are impacted by the specified whatif query and then uses a change propagation technique to propagate the specified workload change through each component in the influence graph.

- Prototype Implementation: We have implemented a prototype of our Predico whatif modeling framework. Our prototype incorporates a What-If Query Language (WIFQL) that can be used by administrators to pose queries. Since our prototype needs to handle large data center applications with hundreds of interacting components, we implement several optimizations to scale the modeling framework to such large applications. Specifically Predico uses on-the-fly model construction and employs a cache of previously constructed models to reduce model computation overhead.

- Evaluation based on real traces and real-world synthetic applications: We conduct an experimental evaluation of Predico using traces of two large production applications from a financial institution as well as realistic synthetic applications. Our experimental results validate the accuracy of Predico's modeling framework in building node-level resource usage, latency and workload models and illustrate Predico's ability to enable accurate what-if analysis.

\section{Background and Problem Formulation}

Our work assumes a large distributed application with $N$ interacting components. We assume that the application is structured as a directed acyclic graph (DAG), where each vertex represents a software component and edges capture the interactions (i.e., flow of requests) between neighboring nodes. For simplicity, we assume that each component runs on a separate physical (or virtual) machine 1 We assume that the DAG has one or more source nodes, that serve as entry points for application requests and one or more sinks, that serve as exits. The flow and processing of requests through such applications is captured by the DAG structure and is best explained with examples.

As examples of such distributed applications we consider two production financial applications. The first application is a stock trade processing application at a major financial firm; the application consists of 471 nodes and 2073 edges. New stock trade requests arrive at one of the source nodes and flow through the system and exit from the sink nodes as "results". Each intermediate node performs some intermediate processing on the trade request and triggers additional requests at downstream nodes. Nodes may aggregate incoming stock trades or break down a large stock order into smaller requests at downstream nodes. Figure 1 shows the structure of a market data dissemination application that disseminates stock prices and news updates for a company to trading terminals ("desktops") of stock traders. In this case, news items arrive from a number of sources and stock prices are obtained from a variety of exchanges, and this

\footnotetext{
${ }^{1}$ This assumption is easily relaxed and we employ it for simplicity of exposition.
} 
information is processed, transformed, filtered and/or aggregated and disseminated to any desktop node that has subscribed to information for a particular company. This application has 8970 nodes and 22719 edges and must provide updates at low latency in order for stock traders to make trades based on the latest market news.

Thus, we assume that requests flow through the DAG, with intermediate processing at each node; a request may trigger multiple requests at one or more downstream child nodes, and each node may aggregate requests from upstream parents. As can be seen, such applications are significantly larger and more complex than traditional multi-tier web applications.

We assume that the DAG structure for each application is known a priori (there are automated techniques to derive the DAG structure by observing incoming and outgoing traffic at each node [8]). We assume that each node in the DAG is a black box-i.e., we can observe the incoming and outgoing request streams along its edges and the total node-level utilization but that we have no knowledge of the internals of the software component and how it processes each request. This is a reasonable assumption in practice since IT administrators typically do not have direct knowledge of the application logic inside a software component, requiring us to treat it as a black box. However, administrators have access to request logs that the application components may generate and can also track OS-level resource utilizations on each node.

We assume that there are $R$ different types of requests in the entire distributed application. Each node can receive different types of requests belonging to the $R$ types and can in turn trigger one or more requests of one of the $R$ types at downstream child nodes. Given our black box assumption, the precise dependence of what type of outputs are generated by what set of inputs is unknown (and must be learned automatically by correlating request logs at a parent and a child). Similarly, the precise processing demands imposed by a set of requests are unknown and must also be learned.

Assuming such a data center application, our first problem is to model each application component (i.e., node of the DAG) by capturing the dependence between the incoming workload mix and the request latency, resource utilization, and the outgoing workload. Second, we need to use these node-level models to create system-level models that capture the behavior of a group of interacting nodes. Third, given such system-level models, we wish to enable rich workload-based what-if analysis of the distributed application. Such an analysis should allow administrators to pose what-if queries to determine the impact of a workload change at a particular node(s) on some other node(s) of the system. A typical what-if query is assumed to contain two parts: (i) the "if" part, which specifies the hypothetical workload change, and (ii) the "what" part, which specifies the nodes where the impact of this change should be computed. For instance, a volume-based what-if query could ask "what is the impact of doubling the volume of requests seen by source node $i$ on the incoming workload and CPU utilization seen at some downstream node $j$ ?" Queries could also be concerned with the impact on latency: "what is the impact of doubling the volume of type $B$ requests at node $j$ on the latency of requests at node $i$ ?"

Thus, to design our what-if analysis system, we must address the following three problems: (i) how should we model the dependence between the incoming workload at a node and the request latency, node utilization and the outgoing workload to 


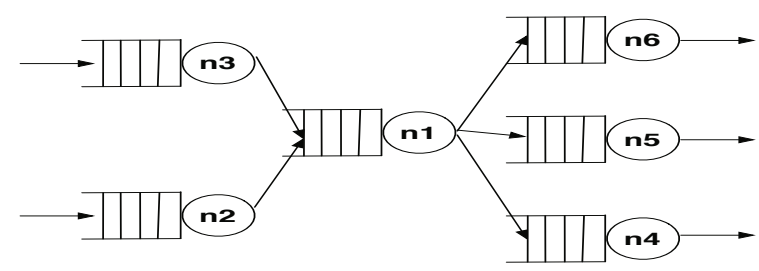

Fig. 2. Modeling a data center application using an open network of queues

downstream nodes? (ii) how should we combine node-level models to create systemlevel models that capture the aggregate behavior of a group of interacting nodes in the DAG? (iii) what algorithms should be used to efficiently execute a what-if query using these models? From an implementation standpoint, we are interested in a fourth question as well: (iv) How should our system scale to complex data center applications with tens or hundreds of components?

\section{Modeling a Data Center Application}

In this section, we first present a queuing model for a data center application that allows us to model the utilization and response time of these nodes. We then describe the construction of models to capture the input/output workload dependencies of these nodes. Finally, we explain how these node-level models are composed to construct systemwide models.

\subsection{Queuing Theoretic Node-Level Models}

Consider the DAG of a data center application with $k$ nodes denoted by $n_{1}, \ldots, n_{k}$ and $R$ different type of requests. We model the data center application using an open network of $k$ queues, one for each node with $R$ classes of requests. We model each node as a $M / G / 1 / P S$ queue i.e. the service times are assumed to have an arbitrary distribution and the service discipline at each node is assumed to be processor sharing (PS). Requests can arrive at a queue from other queues which are its parents in the DAG or in the case of source nodes of the DAG from external sources. For analytical tractability we assume that the distribution of inter-arrival times of requests coming from outside have a poisson distribution. We denote the arrival rates of requests of class $r$ at the queue $n_{i}$ from outside by $\lambda_{0, i}^{r}$. We assume that different classes of requests arriving at a queue have different mean service rates. We denote the mean service rate of requests of class $r$ at node $i$ by $\mu_{i}^{r}$.

Thus the DAG of a data center application is modeled as an open network of queues as shown in Figure 2 We use the well known queueing theory result called the BCMP theorem [1] to analyze this network of queues. The BCMP theorem states that for such queueing networks the utilization, denoted by $\rho_{i}$, of a node $n_{i}$, is given by :

$$
\rho_{i}=\sum_{r=1}^{R} \rho_{i}^{r}=\sum_{r=1}^{R} \frac{\lambda_{i}^{r}}{\mu_{i}^{r}}
$$




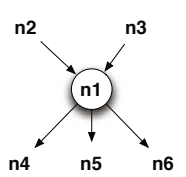

Fig. 3. Node-level model

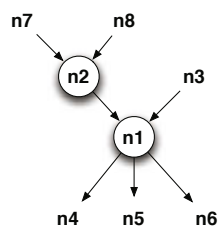

Fig. 4. Model Composition

where $\rho_{i}^{r}$ denotes the resource utilization at node $n_{i}$ due to class $r$ requests, $\lambda_{i}^{r}$ denotes the arrival rate of requests of type $r$ at node $n_{i}$ and $\mu_{i}^{r}$ denotes the service rate of requests of type $r$ at node $n_{i}$. This equation models the resource utilization of the node as a function of the per-class arrival rate and per-class service rates. Similarly, the average number of requests of type $r$ at node $n_{i}$ under steady-state, denoted by $\bar{K}_{i}^{r}$, is given by:

$$
\bar{K}_{i}^{r}=\frac{\rho_{i}^{r}}{1-\rho_{i}}
$$

We can now use Little's Law [3] to find out the $\bar{T}_{i}^{r}$, the average response time of requests of type $r$ at node $n_{i}$ using Equations 1 and 2 .

$$
\bar{T}_{i}^{r}=\frac{\bar{K}_{i}^{r}}{\lambda_{i}^{r}}=\frac{1}{\mu_{i}^{r}\left(1-\rho_{i}\right)}
$$

This equation models the response time at a node as a function of the total node resource utilization $\rho_{i}$ and the per-class service rate $\mu_{i}^{r}$.

Given a value for the per-class workload, $\lambda_{i}^{r}$, at a node we can use Equation 1 to find out the utilization $\rho_{i}$ and then use the computed value $\rho_{i}$ to find out the response time using Equation 3 The per-class service times $\mu_{r}^{i}$ is the only unknown in the equations. Since we assume that each node of the data center application is a black-box we need to estimate these unknowns from the available information gathered from monitoring of the node. We assume that request logs at a node contain an entry for each incoming request containing the timestamp and the request string or type of request and that the resource utilization of the node is being periodically monitored using a tool like iostat. Given such logs, multiple values of $\rho_{i}$ and $\lambda_{i}^{r}$ can be collected over time. Since Equation 1 captures the relationship between these $R+1$ variables, the values of the unknown per-class service rates $\mu_{i}^{r}$ can be numerically estimated using a regression method such as least squares.

\subsection{Workload Models}

While queueing theory allows us to model the performance metrics of a node, we also need to capture the relationship between the incoming workload and the outgoing workload of a node.

To understand the node-level workload models that Predico needs to build, consider the node shown in Figure 3 This node $n_{1}$ has two parent nodes $n_{2}$ and $n_{3}$ and three 
child nodes $n_{4}, n_{5}$ and $n_{6}$. Let $\lambda_{2,1}^{r}$ and $\lambda_{3,1}^{r}$ denote the arrival rate of requests of type $r$ from node $n_{2}$ and $n_{3}$ respectively to node $n_{1}$. Similarly, let $\lambda_{1,4}^{r}, \lambda_{1,5}^{r}$ and $\lambda_{1,6}^{r}$ denote the arrival rate of requests of type $r$ at node $n_{4}, n_{5}$ and $n_{6}$ respectively from node $n_{1}$. Predico needs to build models that capture the workload of each outgoing edge as functions of workload of the incoming edges. Thus, we seek a function for each of $\lambda_{1,4}^{r}, \lambda_{1,5}^{r}$ and $\lambda_{1,6}^{r}$ that expresses them as a function of $\overrightarrow{\lambda_{2,1}}$ and $\overrightarrow{\lambda_{3,1}}$ where $\overrightarrow{\lambda_{i, j}}$ is short-hand for observed rates of various request types on the edge going from node $n_{i}$ to $n_{j}$ i.e. $\left(\lambda_{i, j}^{1}, \lambda_{i, j}^{2}, \cdots \lambda_{i, j}^{R}\right)$. Similarly, we seek functions for each of the other request types:

$$
\lambda_{1,4}^{w}=f_{1,4}^{w}\left(\overrightarrow{\lambda_{2,1}}, \overrightarrow{\lambda_{3,1}}\right) \quad, 1 \leq w \leq R
$$

We model workload-to-workload dependencies as piecewise linear functions. Although these dependencies are linear in steady state by the principle of job flow balance [3], [9], we choose piecewise linear modeling to capture the behavior of caches in servers. For instance, a node with a cache can initially be sending a large number of requests to downstream nodes when the cache is empty, but when the cache becomes full, it might serve requests from its cache instead of sending requests to its downstream nodes. This changing dependence of outgoing workload on incoming workload can be captured by two linear functions, one each for when a cache is cold and and when it is hot. To incorporate piecewise linear modeling, we replace the linear model shown in Equation 4 with a piecewise-linear model by dividing the $2 R$-dimensional space spanned by $\left(\overrightarrow{\lambda_{2,1}}, \overrightarrow{\lambda_{3,1}}\right)$ into $n$ hypercube regions. A linear model is then used to capture the relationship in each of these regions independently. Thus, we can rewrite Equation 4 as a set of linear functions one for each region :

$$
\lambda_{1,4}^{w}= \begin{cases}\sum_{r=1}^{r=R}\left(A_{1}^{w, r} \lambda_{2,1}^{r}+B_{1}^{w, r} \lambda_{3,1}^{r}\right) & \text { if }\left(\overrightarrow{\lambda_{2,1}}, \overrightarrow{\lambda_{3,1}}\right) \in Z_{1} \\ \sum_{r=1}^{r=R}\left(A_{2}^{w, r} \lambda_{2,1}^{r}+B_{2}^{w, r} \lambda_{3,1}^{r}\right) & \text { if }\left(\overrightarrow{\lambda_{2,1}}, \overrightarrow{\lambda_{3,1}}\right) \in Z_{2} \\ \ldots \ldots \ldots \ldots \ldots & \\ \cdots \cdots \cdots & \text { if }\left(\overrightarrow{\lambda_{2,1}}, \overrightarrow{\lambda_{3,1}}\right) \in Z_{n} \\ \sum_{r=1}^{r=R}\left(A_{n}^{w, r} \lambda_{2,1}^{r}+B_{n}^{w, r} \lambda_{3,1}^{r}\right)\end{cases}
$$

where $Z_{i}$ is the $i^{\text {th }}$ hypercube region.

Equation 5 relates the outgoing workload to incoming workload, but to use it for computing the outgoing workload $\lambda_{1,4}^{w}$ for a given value of incoming workload $\overrightarrow{\lambda_{2,1}}$ and $\overrightarrow{\lambda_{3,1}}$ we need to first find the number of regions, $n$, and the regions themselves, $Z_{i}$. We then need to find individual linear functions for each region by computing the weights of the corresponding linear function, $A_{1}^{w, r}$ and $B_{1}^{w, r}$. We use a regression analysis technique called multivariate adaptive regression splines (MARS) [6] that automatically fits piecewise linear functions on data. Predico uses the monitoring data that contains multiple measurements of the variables $\overrightarrow{\lambda_{2,1}}, \overrightarrow{\lambda_{3,1}}$ and $\lambda_{1,4}^{w}$ to give as training data to MARS which finds out the different regions and the linear function in each region.

\subsection{Model Composition: From Node-Level to System-Level Models}

Predico uses node-level models to construct system-wide models using model composition. Model composition essentially "chains" together multiple node-level models to 
compute the workload, resource utilization and response time of a node as a function of one or more ancestor nodes. We illustrate the composition algorithm used by Predico using an example. Consider the sub-graph in Figure 4 that shows a parent node $n_{2}$, extending our earlier example in Figure 3 At the node-level, Predico can compute the outgoing workload going from node $n_{2}$ to node $n_{1}, \overrightarrow{\lambda_{2,1}}$, as a set of $R$ piecewise linear functions, one for each request type :

$$
\lambda_{2,1}^{w}=f_{2,1}^{w}\left(\overrightarrow{\lambda_{8,2}}, \overrightarrow{\lambda_{7,2}}\right) \quad, 1 \leq w \leq R
$$

Equation 4 gives the outgoing workload going from node $n_{1}$ to $n_{4}$ :

$$
\lambda_{1,4}^{w}=f_{1,4}^{w}\left(\overrightarrow{\lambda_{2,1}}, \overrightarrow{\lambda_{3,1}}\right) \quad, 1 \leq w \leq R
$$

Substituting the value of $\overrightarrow{\lambda_{2,1}}$ from Equation 6 into Equation 7 we obtain a "composed model":

$$
\lambda_{1,4}^{w}=f_{1,4}^{w}\left(\overrightarrow{f_{2,1}}\left(\overrightarrow{\lambda_{8,2}}, \overrightarrow{\lambda_{7,2}}\right), \overrightarrow{\lambda_{3,1}}\right) \quad, 1 \leq w \leq R
$$

where $\overrightarrow{f_{2,1}}\left(\overrightarrow{\lambda_{8,2}}, \overrightarrow{\lambda_{7,2}}\right)$ is shorthand for $\left(f_{2,1}^{1}\left(\overrightarrow{\lambda_{8,2}}, \overrightarrow{\lambda_{7,2}}\right), f_{2,1}^{2}\left(\overrightarrow{\lambda_{8,2}}, \overrightarrow{\lambda_{7,2}}\right), \ldots\right.$, $\left.f_{2,1}^{R}\left(\overrightarrow{\lambda_{8,2}}, \overrightarrow{\lambda_{7,2}}\right)\right)$. Doing so enables the outgoing workload sent from node $n_{1}$ to $n_{4}$ to be expressed as a function of incoming workload of parent node $n_{2}$. This process can be repeated for the outgoing workload going to nodes $n_{5}$ and $n_{6}$ from node $n_{1}$ and can also be recursively extended to nodes that are further upstream from $n_{2}$.

Creation of the composed model shown in Equation 8 requires composing the piecewise linear function $f_{1,4}^{w}$ with each of the $R$ piecewise linear functions $f_{2,1}^{w}, 1 \leq w \leq R$. Two piecewise linear functions can be easily composed by composing the individual linear functions in each corresponding region which leads to another piecewise linear function. Thus the composed model shown in Equation 8 is again a piecewise linear function which captures the relation between the outgoing workload of node $n_{1}$ and the incoming workload of a parent node $n_{2}$.

We can now do a similar composition to find the dependence of the resource utilization of node $n_{1}$, denoted by $\rho_{1}$, and the response time of requests of type $r$ at node $n_{1}$, denoted by $\bar{T}_{1}^{r}$ on the incoming workload of parent node $n_{2}$ denoted by $\overrightarrow{\lambda_{8,2}}, \overrightarrow{\lambda_{7,2}}$. Substituting Equation 6 into the resource utilization equation given by Equation 1 we get :

$$
\begin{aligned}
\rho_{1} & =\sum_{r=1}^{R} \rho_{1}^{r}=\sum_{r=1}^{R} \frac{\lambda_{1}^{r}}{\mu_{1}^{r}}=\sum_{r=1}^{R} \frac{\lambda_{3,1}^{r}+\lambda_{2,1}^{r}}{\mu_{1}^{r}} \\
& =\sum_{r=1}^{R} \frac{\lambda_{3,1}^{r}+f_{2,1}^{r}\left(\overrightarrow{\lambda_{8,2}}, \overrightarrow{\lambda_{7,2}}\right)}{\mu_{1}^{r}}
\end{aligned}
$$

which expresses the resource utilization of node $n_{1}$ as a function of the incoming workload of node $n_{2}$. Similarly, we can substitute from Equation 10 into the response time Equation 3 to express the response time of request type $r$ at node $n_{1}$ as a function of the incoming workload of parent node $n_{2}$ : 


$$
\bar{T}_{1}^{r}=\frac{1}{\mu_{1}^{r}\left(1-\rho_{1}\right)}
$$

\section{Answering What-if Queries}

In this section we describe the three step process used by Predico to answer a given what-if query. The execution of a what-if query is a three step process comprising of: 1) finding the influence graph of the given query, 2) creating the node-level models of the nodes in the influence graph using the modeling technique described above and 3) using the change propagation algorithm to execute the query. We describe the three steps in greater detail below.

\subsection{On-the-Fly Model Construction Using the Influence Graph}

Since the number of nodes and edges in the DAG may be large in complex applications, it is not economical to precompute all possible node-level models and periodically recompute models that have become invalid due to an actual workload or hardware change. Instead Predico employs a "just-in-time" policy to compute models on-the-fly when a query arrives; only those models that are necessary to answer the query are computed. Models from prior queries are cached and reused if they are still valid. Predico uses the notion of an influence graph to determine which models should be constructed to answer a query. Given a what-if query, the influence graph is the set of all possible paths from the nodes in the "if" part of the query to the nodes in the "what" part. Basically the influence of a workload change will propagate along all paths from the "if" nodes/edges to the "what" nodes; so the influence graph captures all of the nodes that must be considered to answer the query and other nodes in the DAG can be ignored.

Upon the arrival of a what-if query, Predico first computes the influence graph by generating the set of nodes that lie along all paths from the "if" nodes/edges to the "what" nodes. It then triggers on-demand construction of node-level workload models for all the nodes in the influence graph and node-level resource utilization and response time models for the "what" nodes alone. The use of the influence graph to prune the DAG and the reuse of previously computed models from the model cache enhances the scalability of the system and reduces computational overheads. The influence graph is also crucial for efficient query execution, as we will see in the next section.

\subsection{Query Execution Using Change Propagation}

After creating the node-level models for the nodes of the influence graph, Predico now needs to "execute" the query. Query execution involves propagating the specified workload change through the influence graph, one node at a time, to compute its final impact on the nodes/edges specified in the "what" part of the query. Once the workload change has been propagated to the nodes in the "what" part, the node-level models can be used to answer the query. Change propagation is equivalent to model composition-instead of directly computing a composed model for the "what" nodes/edges as a function of the 


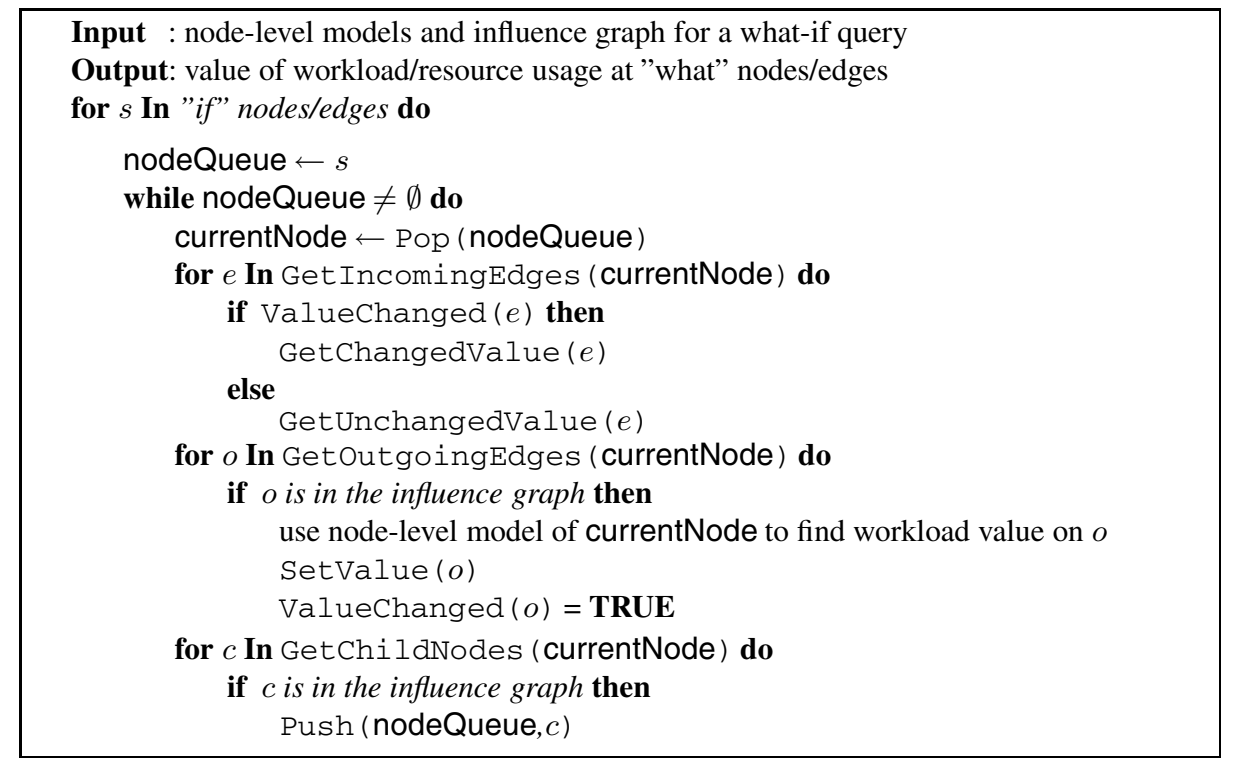

Algorithm 1. Change Propagation via the Influence Graph

"if" nodes/edges, the propagation algorithm propagates the specified change through the influence graph all the way down to the nodes/edges in the "what" part to achieve the same result.

Predico's change propagation algorithm is described in Algorithm 1 It takes the node-level models and the influence graph, and traverses the influence graph in a breadth first manner. It starts with the nodes/edges in the "if" part and computes the values for the changed workload and then uses the model to compute its impact on the outgoing workload. This process is referred to as propagating the change from the incoming edges of a node to its outgoing edges. To illustrate, consider a query that is interested in estimating the impact of a doubling of the workload for a particular edge. If the original request rate was $10 \mathrm{req} / \mathrm{s}$, then the new workload will be $20 \mathrm{req} / \mathrm{s}$ for that edge. This new value is used, along with the unchanged request rates for all other edges not impacted by the change, to compute the outgoing request rates for that node.

The algorithm proceeds in a breadth first fashion through the influence graph, starting with the "if" nodes/edges and computing the outgoing workload for each of the "if" nodes. The outgoing workload of a node becomes the incoming workload for downstream node(s), and the change propagation process repeats, one node at a time, in a breadth-first fashion, until the change has propagated to all of the "what" nodes/edges. At this point, the algorithm computes the value of interest at the node by using the node-level models and terminates.

\section{Predico Implementation}

This section describes WIFQL, a query language that can be used to pose what-if queries to Predico and the implementation details of Predico prototype. 


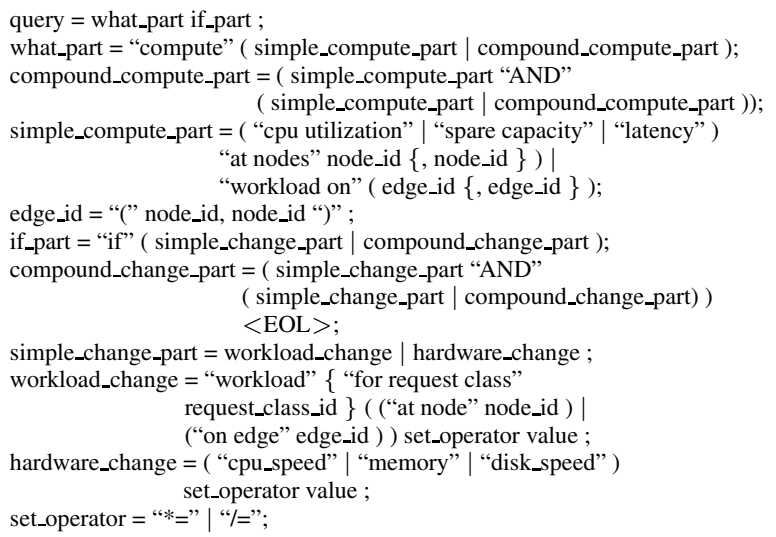

Fig. 5. The grammar for Predico's What-If Query Language (WIFQL)

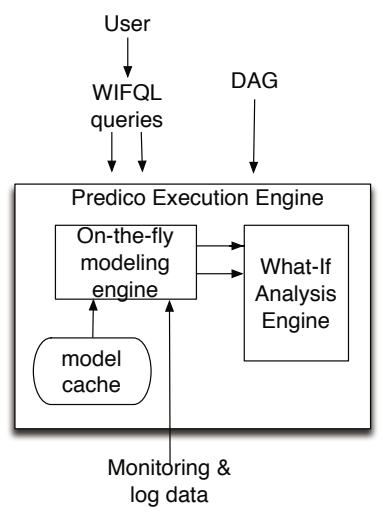

Fig. 6. Predico Architecture

\subsection{Posing What-if Queries in Predico}

Since the goal of Predico is to enable users to understand the impact of potential workload changes on the system behavior, our system supports a simple query language to enable a rich set of queries to be posed by IT administrators. Any query in our What-If Query Language (WIFQL) has two parts: a what part and an if part. The if part of the query describes the hypothetical change, while the what part asks the system to compute the impact of that change on different performance metrics at one or more nodes in the system. As an example of an WIFQL query, consider

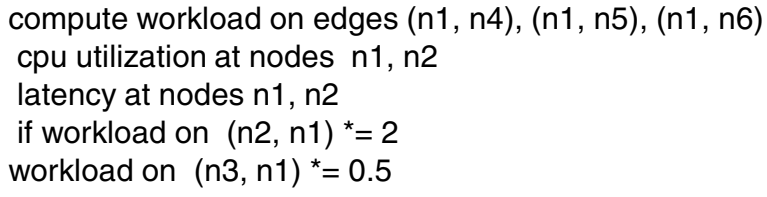

This example query asks the system to compute the impact of a doubling of the workload along the edge going from node $n_{2}$ to $n_{1}$ and a halving of the workload along the edge going from node $n_{3}$ to $n_{1}$ on the CPU utilization and latency at nodes $n_{1}$ and $n_{2}$ and the workload on the edges going from node $n_{1}$ to nodes $n_{4}, n_{5}$ and $n_{6}$.

Figure 5 describes our query language grammar. As shown, the if part allows users to specify hypothetical changes to the workload or changes to the hardware (e.g., a faster $\mathrm{CPU}$ ). The workload changes, which is the focus of this work, can be specified by identifying one or more edges or nodes in the DAG and indicating a change in volume or a change in the mix of requests; set operators such as multiply and divide can be used to specify relative changes to the current workload, rather than absolute values. The what part specifies the performance metrics of interest at particular nodes or edges; several 
metrics are supported including resource utilizations, workloads, latencies or spare capacities. As indicated earlier, we assume that the DAG representing the application is known a priori and is used by queries to refer to particular nodes and edges of interest and specify workload changes on these nodes or edges.

\subsection{Prototype Implementation}

We have implemented a prototype of Predico using Python and the R statistical language to perform what-if analysis in large data center applications. Figure 6 depicts the high-level architecture of Predico.

The Predico frontend is implemented using a python implementation of the lex and yacc parsing tools. It accepts user-posed queries and parses them by using the grammar rules of WIFQL. User-posed queries are then executed by the Predico execution engine, which comprises of two key components; the on-the-fly modeling engine and the what-if analysis engine. The on-the-fly modeling engine first computes the influence graph using a graph API in python and then creates node-level models by using on-the-fly model construction. The modeling engine retrieves data about the workload on the incoming and outgoing edges of the node and the total resource utilization of the node and then invokes an $\mathrm{R}$ module for building the node-level models. The $\mathrm{R}$ module uses the MARS function present in the MDA package to build piecewise linear nodelevel workload models and the linear regression function to find the per-class service rates using least squares regression. Next, the what-if analysis engine uses these models to answer ("execute") the query via the change propagation algorithm to propagate the hypothetical workload change through the model and compute its impact on the nodes of interest to the user. The change propagation algorithm is again implemented by using the graph API written in python. The what-if analysis engine stores the node-level models computed by the modeling engine in a model cache that is implemented as three tables in the MySQL relational database engine; one each for storing the weight vectors used in node-level workload models, the regions of the piecewise-linear model and the per-class service rates of a node required in the node-level resource utilization and response-time models.

\section{Experimental Evaluation}

In this section, we evaluate the performance of Predico by performing experiments on two applications. We first evaluate the accuracy of the analytical node-level resource utilization and response-time models and then the piecewise-linear workload models. We then perform experiments to ascertain the accuracy of system-level models formed by composition. We then employ Predico to perform case studies where we pose whatif queries to Predico and compare the predictions with ground truth values observed in actual experimental data.

\subsection{Experimental Setup}

We evaluate Predico on two different applications. The first set of applications are from the financial domain and are being used by the data center of a financial institution. The second application is a benchmark e-commerce application. 
1. We evaluate our system on traces collected from the two production financial applications described in Section 2 . The traces collected from the stock trade processing application contain the total number of requests sent out by every component within every 30 second interval. The traces collected from the market data dissemination application contain data for the number of bytes sent out from every component on each of its outgoing edge, within every 30 second interval. Table 1 lists the characteristics of the traces.

Table 1. Characteristics of Production Traces

\begin{tabular}{|c|c|c|c|c|c|}
\hline Application & \#Nodes & $\#$ Edges & Duration & Metric & \# of Records \\
\hline $\begin{array}{c}\text { Market Data } \\
\text { Dissemintation }\end{array}$ & 8970 & 22719 & 1 day & outgoing bytes & 7763764 \\
\hline $\begin{array}{c}\text { Stock Trade } \\
\text { Processing }\end{array}$ & 471 & 2073 & 4 days & outgoing requests & 6060952 \\
\hline
\end{tabular}

2. The second application is the TPC-W benchmark [15] which models an online bookstore application. We implement the TPC-W application as a 2-node Java servlet based application consisting of the front-end server (Tomcat) and a backend database (MySQL). Notice that this application does not follow our DAG assumption since replies are sent back from the back-end database to the front-end server. Predico is also able to handle such applications that contain cycles between neighboring nodes by considering the two edges of the cycle separately. To implement this application we use a testbed comprising of two virtual machines for performing this experiment. Each virtual machine has a single $2.8 \mathrm{GHz}$ Pentium 4 processor with $1 \mathrm{~GB}$ memory. We use Tomcat version 5.5.26 and MySQL version 5.1.26 for setting up our TPC-W application. The TPC-W experimental setup allows us to monitor the end-to-end latency and resource utilization values apart from workload values.

\subsection{Accuracy of Node-Level Resource Usage and Latency Models}

We model the data center application as an open network of queues that lead to Equation 1 which captures the node-level resource utilization and Equation 3 which captures the node-level latency. We validate the accuracy of this queueing model using the TPCW application running on a two server testbed.

We use the httperf load generation tool to simulate requests arriving from customers with exponentially distributed inter-arrival times. The TPC-W web application exposes 14 different servlets which a customer visiting the website can invoke. We create a workload comprising of requests to two of these servlets, the "new products" and "execute search". We independently vary the arrival rate of requests to both these servlets from 10 to 100 requests per second with increase of 10 requests per second, thus generating a total of 100 arrival rate combinations. For each arrival rate combination, we let the system run for 15 minutes and measure the CPU utilizations at the Tomcat and MySQL server and the end-to-end latency. We use half of the 100 values for estimating 


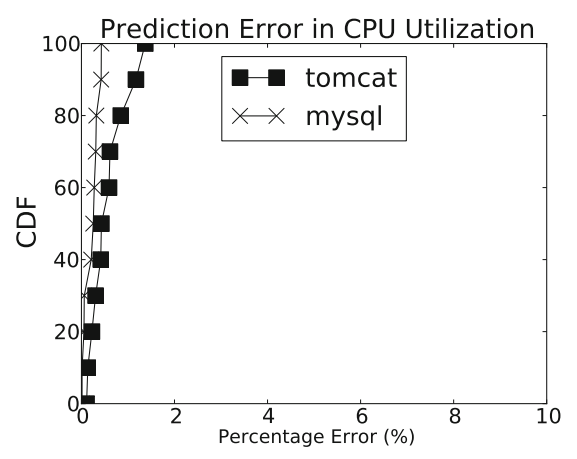

Fig. 7. Node-level Resource Usage model Accuracy

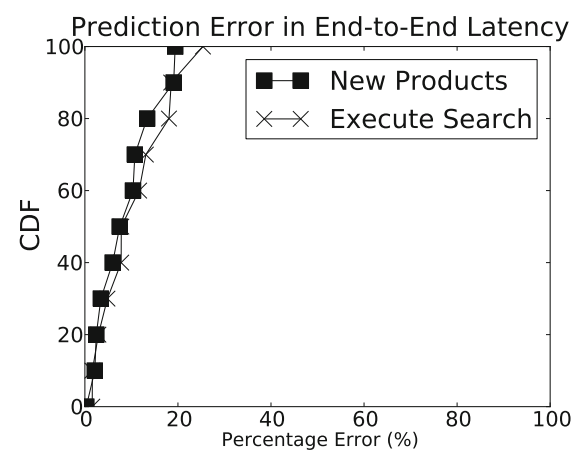

Fig. 8. Node-level Latency model Accuracy

the values of the per-class service rates on each of the 2 nodes and then use these values to predict the per-node resource utilizations and per-node per-class response time for the other half. The per-node response times are summed up to get the end-to-end latency. We compute the prediction errors by comparing the predictions of the node-level models with the values observed during the experiments. Figure 7 and Figure 8 shows the distribution of prediction errors in terms of percentage relative error in predicting the resource utilization and latency respectively.

By using an open network of queue modeling, we are able to predict node-level CPU utilization to within $2 \%$ of the actual value. The median prediction error for response time using our modeling approach is less than $10 \%$.

\subsection{Accuracy of Node-Level Workload Models}

We evaluate the accuracy of using piecewise-linear functions created by using MARS to model the relationship of the outgoing workload of a node with the incoming workload of the node. We use the traces collected from the two applications to create these models and then ascertain the accuracy of these models.

For each of the two applications, we selected each component in turn and extracted the data for the workload on its incoming edges and outgoing edges. We then use MARS to estimate a function which expresses the workload on each outgoing edge of a component as a piecewise linear function of the workload on all the incoming edges on the component. We evaluate the accuracy of the piecewise linear model in predicting the workload on each outgoing edge of this component. Cross-validation was used to measure the prediction accuracy; we divide the trace data for the selected component into training windows of 1 hour each and compute a model using MARS for each window for each outgoing edge. We then use each model to predict the data points outside of the window it was trained on; the deviations between the predicted and actual values were measured. We use the root mean square (RMS) error as a metric of error; we divide the RMS error by the range of actual values to report the results in normalized RMS error (\%). The average normalized RMS error for the models of all the outgoing edges of a component is taken as the error for that component. We depict the errors for all 


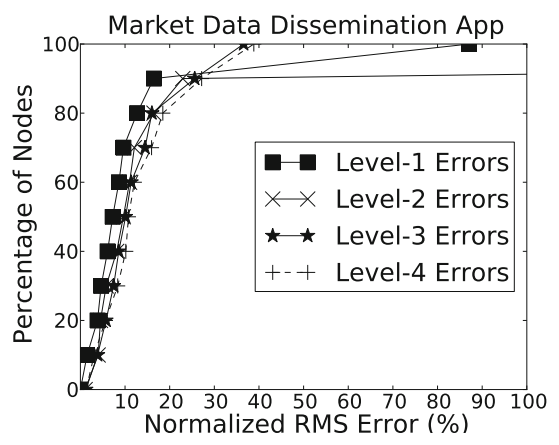

Fig. 9. Composed modeling for Market Data Dissemination App

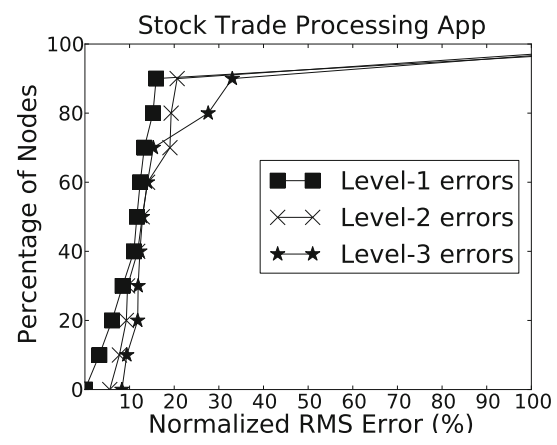

Fig. 10. Composed Modeling for Stock Trade Processing App

the components of the two applications using CDF curves that show the percentage of components that have errors below a certain value. Figure 9 shows the errors for the market data dissemination application while Figure 10 shows the errors for the stock trade processing application. The curve labeled "Level-1" errors shows the CDF for the errors. We describe the concept of levels and the description about the "Level-2" and "Level-3" curves later in this section. The CDF curves indicate that the workload-toworkload models of $70 \%$ of the components have errors less than $10 \%$ in the case of the market data dissemination application while models for $80 \%$ of the components have errors less than $15 \%$ in the case of the stock trade processing application.

Our experimental results show that piecewise linear modeling provides accurate models of node-level workload for production data center applications.

\subsection{Accuracy of System-Level Models with Increasing Composition Depth}

We evaluate the accuracy of system-level models created by composing multiple nodelevel models. Composition of multiple node-level models leads to an accumulation of the error terms. We conduct experiments to measure the increase in error with composing increasing number of node-level models. We again use the traces from the two financial applications to evaluate the accuracy of system-level models. We reuse the node-level models of each component built for validating the accuracy of node-level workload models in the previous section for this experiment.

We select each component and compose its node-level workload model with that of its ancestor components to express the outgoing workload of this component as a function of the incoming workload of its ancestors. By using composition repeatedly we successively construct models expressing workload of a component as a function of its ancestors at different levels. Level 1 model is built between the outgoing workload of a component and its incoming workload. Level 2 model is built between the outgoing workload of a component and the incoming workload of its immediate parents. Similarly level $i$ model is built between the component and its ancestors that are reachable in $(i-1)$ edges. We compute the average normalized RMS error of each component by computing the average normalized RMS error for the models of all the outgoing edges 


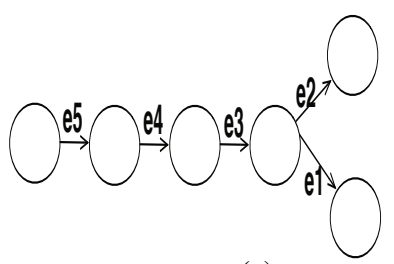

(a)

\begin{tabular}{|c|c|c|c|c|}
\hline Prediction Source & \multicolumn{4}{|c|}{ Prediction Target } \\
\hline & $\mathrm{e} 1$ & $\mathrm{e} 2$ & $\mathrm{e} 3$ & $\mathrm{e} 4$ \\
\hline $\mathrm{e} 3$ & $2.4 \%$ & $2.6 \%$ & & \\
\hline $\mathrm{e} 4$ & $4.7 \%$ & $3.05 \%$ & $4.39 \%$ & \\
\hline $\mathrm{e} 5$ & $4.67 \%$ & $2.95 \%$ & $4.33 \%$ & $0.79 \%$ \\
\hline
\end{tabular}

(c)

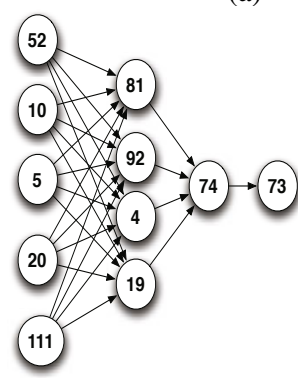

(b)

\begin{tabular}{|c|c|c|c|c|c|c|}
\hline $\begin{array}{c}\text { Prediction } \\
\text { Source }\end{array}$ & \multicolumn{5}{|c|}{ Prediction Target } \\
\hline & 81 & 19 & 74 & 73 & 4 & 92 \\
\hline $111,20,5,101,52$ & $12.47 \%$ & $12.45 \%$ & $10.2 \%$ & $9.28 \%$ & $12.5 \%$ & $12.54 \%$ \\
\hline $19,4,92,81$ & & & $10.63 \%$ & $9.46 \%$ & & \\
\hline 74 & & & & $3.34 \%$ & & \\
\hline
\end{tabular}

(d)

Fig. 11. Prediction Errors of composed modeling on different topologies

of the component using cross-validation and then averaging the errors. Figures 9 and 10 show the CDF of normalized RMS errors for each level for the two applications. The CDF curve drops with increasing levels implying that the errors increase as we predict the workload of a component using ancestors higher up the component in the graph. Inspite of the increasing errors with increasing levels, the errors remain tolerable; for the Market Data Dissemination application even at level 4 the prediction errors for $80 \%$ of the components are less than $20 \%$, while for the Stock Trade Processing at the level of 3 for $75 \%$ of the components the errors are less than $20 \%$.

Our results on using composition to create system-level models on the traces collected from the two production applications reveal that even with increasing composition depth, the system-level models are effective in predicting workload.

\subsection{Accuracy of System-Level Models with Varying Topology}

The node-level models can be composed in a number of ways to create a system-level model depending on the topology of the DAG. We perform experiments to ascertain the prediction accuracy of composed models under different topologies. For this experiment we select some subgraphs in the DAGs for the two applications. We select subgraphs that correspond to three topologies-chain, split and join. These topologies correspond to different ways in which the components can interact with one another in an application: (i) in the chain topology, each component receives requests from a single upstream component, (ii) in the split topology, a component can send requests to multiple downstream components and (iii) in the join topology, a component can receive requests from multiple upstream components. For each subgraph, we create node-level models for each component and then use composition to create models to predict the workload on each outgoing edge of the subgraph. We measure prediction 


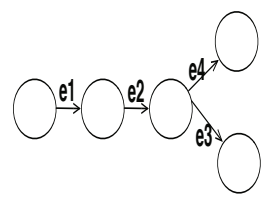

\begin{tabular}{|c|c|}
\hline $\begin{array}{c}\text { Prediction } \\
\text { Hour }\end{array}$ & \\
\hline & $\mathrm{e} 1$ \\
\hline$h 1$ & $A$ \\
\hline$h 2$ & $2 A$ \\
\hline$h 3$ & $2.5 A$ \\
\hline
\end{tabular}

(a) Subgraph 1

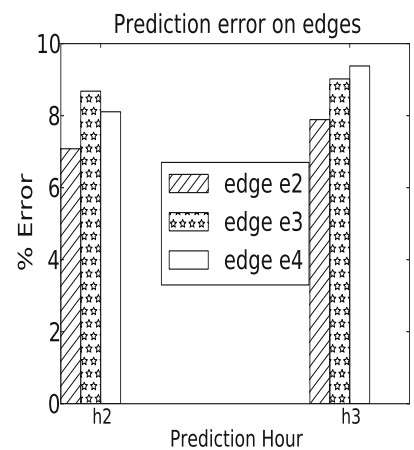

(c) Prediction Errors

Fig. 12. What-If case study on Market Data Dissemination Application

errors in predicting workload of each outgoing edge as a function of incoming workload of its ancestors at increasing levels.

Figures 11 a) and 11 (b) show the subgraphs that we choose for this experiment. Figures 11 (a) is from the Market Data Dissemination application and Figure 11 (b) is a subgraph from the Stock Trade Processing application. Figure 11 (a) illustrates the chain and split topologies, while figure 11(b) is an example of a join and split topology. Tables 11 (c) and 11 (d) show how the errors of the composed models vary as we predict the workload on various edges/nodes of the graphs. For the subgraphs selected from the market data dissemination application the prediction errors on all edges are within $5 \%$ while for the subgraph selected from the stock trade processing application the prediction errors are within $13 \%$.

The errors reveal that Predico's composition based modeling technique performs well even in case of complex application topologies.

\subsection{Workload-Only What-If Analysis Case Study}

We create use-case scenarios to illustrate how Predico can be used in practice and evaluate its performance in answering what-if questions which commonly arise in large-scale applications. In this section, we pose workload-related what-if questions; we choose subgraphs from the market data dissemination application and the stock trade processing application and use Predico to predict the impact of workload changes on source nodes at the workload on the other edges of the subgraphs.

We choose one subgraph each from the market data dissemination application and the stock trade processing application. The first subgraph has 1 source node while the other subgraph has 3 source nodes.

The topology of the first subgraph is shown in Figure 12 (a). On this subgraph we pose the query: "what happens to the workload on downstream edges of subgraph 1 if the outgoing workload of the single source node increases by 2 and 2.5 times the current value". We examine the application traces and find periods of 1 hour duration each, $h_{1}$, $h_{2}$ and $h_{3}$, such that the outgoing workload from the source node increases by 2 and 2.5 times the workload in $h_{1}$ in the hours $h_{2}$ and $h_{3}$ respectively. Predico uses the trace 


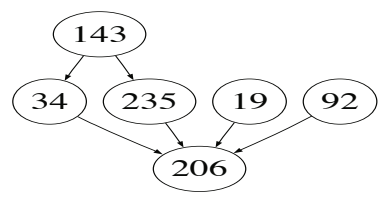

\begin{tabular}{|c|c|c|}
\hline $\begin{array}{c}\text { Prediction } \\
\text { Hour }\end{array}$ & \multicolumn{2}{|c|}{ Node } \\
\hline & 143 & 19,92 \\
\hline h1 & A & B \\
\hline h2 & $1.12 \mathrm{~A}$ & $1.5 \mathrm{~B}$ \\
\hline h3 & $1.8 \mathrm{~A}$ & $1.65 \mathrm{~B}$ \\
\hline
\end{tabular}

(a) Subgraph 2

(b) Subgraph 2 Average Workload

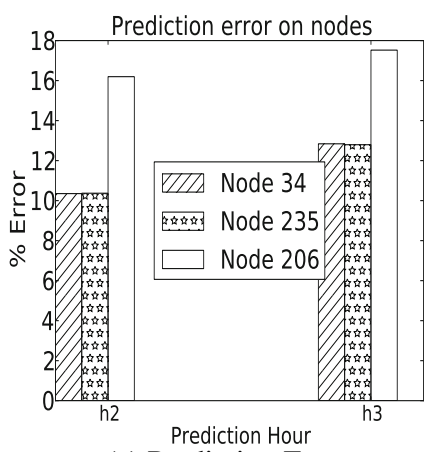

(c) Prediction Errors

Fig. 13. What-If case study on Stock Trade Processing Application

from hour $h_{1}$ and then predicts the workload values in hours $h_{2}$ and $h_{3}$. We compare the ground truth value of the workload seen in the two hours and compare Predico's predictions to compute the errors. Figure 12 (c) plots the errors on all the downstream edges in terms of the normalized RMS error for each of the two changes mentioned in the what-if question.

The topology of the second subgraph is shown in Figure 13 a). On this subgraph we pose the query: "what happens to the workload on downstream nodes a) if the workload on the source nodes 143, 19 and 92 becomes 1.12, 1.5 and 1.5 times respectively the current value $b$ ) if the workload on the source nodes 143, 19 and 92 becomes 1.8, 1.65 and 1.65 times respectively the current value times". We compare Predico's prediction with ground truth values observed in the traces to compute the errors. Figure 13 (c) plots the errors for the two queries for the downstream nodes in terms of normalized RMS error.

The trace collected from the stock trade processing application only contain the requests going out of each node and we assume that these requests are equally distributed among all its outgoing edges. Similarly, in the case of the market data dissemination application, the traces contain the bytes sent out on each edge and we assume that the number of bytes are an approximation of the number of requests. We note that even under these simplifying assumptions, Predico is able to make predictions with errors between $8 \%$ and $18 \%$.

\section{Related Work}

A number of recent efforts have focused on building systems for performing whatif analysis on various distributed systems. The design and implementation of a selfpredicting cluster-based storage system is presented in [13]. The approach, however, involves intrusive instrumentation of the system that is not feasible in production environments. WISE [12] is a system for answering what-if deployment and configuration questions for content distribution networks (CDN). WISE, however, only answers questions related to network latency and does not consider the server processing within data centers. 
Apart from systems that are directly aimed at performing what-if analysis, a number of modeling techniques have been proposed that predict the performance of the system and can be employed for answering what-if questions about the system. Most of these techniques are aimed at multi-tiered systems. A number of these techniques use queuing models to predict the response time and resource utilization of such applications [16],[2],[5]. Similar to our approach, least squares is used to parameterize the queuing models in [17]. Similar to our automatic model derivation, the authors of [7] also automatically derive node-level models to capture relationships between workload; their technique is based on linear models while we have used a queuing-network modeling based approach. In [10], nonstationarity in workloads is utilized to derive models for predicting the resource utilization and response time of an application as a function of workload volume and workload mix. The modeling approach proposed in [11] creates "profiles" for the different components of a distributed application to model the resource demands placed by the components under different workloads on the underlying hardware. IRONModel [14] proposes a modeling architecture for creating robust models. The models are used for answering what-if questions about the impact of reconfigurations on the response time and throughput of a large storage system. In contrast to these systems, Predico is aimed at large-scale systems and enables easily modeling arbitrary distributed applications by joining together individual node-level models. Modellus [4] also uses composition of models to model data center applications. Modellus, however, models workload-to-workload interactions only while Predico looks at response time models as well. Also, Modellus is only a modeling framework, while Predico combines modeling with a full-scale what-if analysis system.

\section{Conclusions}

Data center operators often need to ascertain the impact of unseen workload changes on large distributed applications. Predicting how a certain change in workload will influence complex data center applications is a challenging problem that needs automation. In this paper we presented Predico, a system which enables the user to perform "what-if" analysis on large distributed applications. Predico is non-intrusive and only uses commonly available monitoring data to construct models and uses a new change propagation technique to estimate the impact of specified workload changes.

We modeled a large-scale data center application as an open network of queues to derive resource utilization, latency and workload models. We used traces from two large production applications from data centers of a major financial institution and data from synthetic enterprise applications to evaluate the efficacy of Predico's what-if modeling framework. Our experimental evaluation validated the accuracy of the node-level resource utilization, response time and workload models and then showed how Predico enables what-if analysis in two different applications.

Acknowledgements. This reseach was supported in part by NSF grants CNS-0855128, CNS-0916972, CNS-0720616 and OCI-1032765. 


\section{References}

1. Baskett, F., Mani Chandy, K., Muntz, R.R., Palacios, F.G.: Open, closed, and mixed networks of queues with different classes of customers. J. ACM 22(2), 248-260 (1975)

2. Bennani, M.N., Menascé, D.A.: Resource allocation for autonomic data centers using analytic performance models. In: ICAC, Washington, DC, USA, pp. 229-240 (2005)

3. Denning, P.J., Buzen, J.P.: The operational analysis of queueing network models. ACM Comput. Surv. 10, 225-261 (1978)

4. Desnoyers, P., Wood, T., Shenoy, P., Patil, S., Vin, H.: Modellus: Automated Modeling of Complex Internet Data Center Applications. Technical report, UMass CS (2009)

5. Diao, Y., Hellerstein, J.L., Parekh, S.S., Shaikh, H., Surendra, M., Tantawi, A.N.: Modeling differentiated services of multi-tier web applications. In: MASCOTS, pp. 314-326 (2006)

6. Friedman, J.H.: Multivariate adaptive regression splines. The Annals of Statistics 19(1) (1991)

7. Jiang, G., Chen, H., Yoshihira, K.: Discovering Likely Invariants of Distributed Transaction Systems for Autonomic System Management. In: ICAC, Dublin, Ireland, pp. 199-208 (June 2006)

8. Kind, A., Hurley, P., Massar, J.: A Light-Weight and Scalable Network Profiling System. ERCIM News 60 (2005)

9. Menascé, D.A., Almeida, V.A.F.: Capacity planning for Web performance: metrics, models and methods. Prentice-Hall, Inc., Upper Saddle River (1998)

10. Christopher, S., Terence, K., Alex, Z.: Exploiting nonstationarity for performance prediction. In: EuroSys, pp. 31-44 (2007)

11. Stewart, C., Shen, K.: Performance Modeling and System Management for Multi-component Online Services. In: Proc. USENIX Symp. on Networked Systems Design and Implementation (NSDI) (May 2005)

12. Tariq, M., Zeitoun, A., Valancius, V., Feamster, N., Ammar, M.: Answering what-if deployment and configuration questions with wise. SIGCOMM Comput. Commun. Rev. 38(4), 99-110 (2008)

13. Thereska, E., Abd-El-Malek, M., Wylie, J.J., Narayanan, D., Ganger, G.R.: Informed data distribution selection in a self-predicting storage system. In: ICAC, pp. 187-198. IEEE Computer Society, Washington, DC (2006)

14. Thereska, E., Ganger, G.R.: Ironmodel: robust performance models in the wild. SIGMETRICS Perform. Eval. Rev. 36(1), 253-264 (2008)

15. TPC. The tpcw benchmark, http://www.tpc.org/tpcw/

16. Urgaonkar, B., Pacifici, G., Shenoy, P., Spreitzer, M., Tantawi, A.: An Analytical Model for Multi-tier Internet Services and Its Applications. In: Proc. of the ACM SIGMETRICS Conf., Banff, Canada (June 2005)

17. Zhang, Q., Cherkasova, L., Smirni, E.: A regression-based analytic model for dynamic resource provisioning of multi-tier applications. In: ICAC, Washington, DC, USA (2007) 